\title{
Reflections on the open abdomen
}

\section{Reflexões sobre o abdome aberto}

\author{
Domingos André Fernandes Drumond, TCBC-MG¹.
}

$T^{\mathrm{n} o m}$ he Damage Control Surgery required from the surgeon a paradigm shift, which previously aimed at the definitive treatment of injuries and closure of the abdominal cavity at all costs. The abdominal compartment syndrome, unappreciated and belatedly recognized until then, was certainly the catalyst factor of bad evolution of many trauma patients. Leaving the abdomen open in damage control surgery reignited the importance of the compartment syndrome and softened the impact of this occurrence. It is the good side of the story.

The damage control, a good expression coined by Rotondo et al. ${ }^{1}$, in 1993, and borrowed from the US Navy, was seen as a simple resource, performed by any surgeon at any hospital. From the results and documented experience, we see that it is not so. Damage control and open abdomen are not as simple as they look decision. We enter the mature phase of performing these procedures, but there is a lot of learning ahead.

There is no denying the importance of liver bleeding control through liver packing. It is also understandable to be fast to control bleeding and contamination of the agonized patient. The benefit of scheduled laparotomies also seems clear in unstable patients with multiple injuries and too prolonged surgical time to definitely correct all injuries.

Unfortunately, the range of indications of scheduled surgery and open abdomen has increased. For reasons previously not seen, one interrupts the operation to finish it later. It is not easy to follow the protocol of damage control / laparostomy: there is too much subjectivity in the procedure's indication. Laboratory documentation and monitoring are often insufficient. The surgeon decides to suspend the procedure not because he/she cannot execute it, but for often inconsistent reasons.
Damage control / laparostomy is not an easy decision strategy. Besides the severity of cases, treatment success depends on the performance of the surgical team and on the involvement of anesthesiologists and intensivists. It also depends on material resources and, as an orchestra, there is a system involving professionals imbued with the purpose of masterly performing a complex symphony.

These patients do not tolerate misconceptions and improvisations. The window for the definitive treatment, when opened, is short. Otherwise, the inflammatory response takes control of the scene and compromises the final surgical outcome.When the conventional abdomen closure is not possible in the fourth stage of the damage control procedure (definitive surgery), a difficult and painful task begins, consisting in keeping under protection the viscera and the very abdominal wall, through resources not always the most suitable.

The Bogota bag should not be the unique resource for the open abdomen. It provides for alternative closing between the second and fourth stages. Thereafter, should the abdomen remain without the permanent closure, one should employ dressing with negative pressure whenever possible. It is more reasonable.

The entero-atmospheric fistula is a serious complication of laparostomy. Treatment is difficult, sometimes challenging. Bowel loops without the natural protection of the abdominal wall are vulnerable to perforation for a number of reasons and the Bogota bag is the one with higher fistula incidence. No less difficult to deal with are the other complications of the open abdomen: bowel obstruction, abscesses, intestinal fistulas, incisional hernias.

Accordingly, morbidity and mortality are significant in patients with multiple injuries admitted in critical condition. What concerns, in fact, it is the trivialization of the operative strategy and the interruption

1 - Department of General Surgery and Trauma, João XXIII Hospital, Minas Gerais Hospital Foundation, Belo Horizonte, MG, Brazil. 
of the abdominal surgery without proper assessment of the consequences of that decision.

Are we facing a new iatrogeny in trauma?

In trauma services, it seems appropriate to review protocols on abbreviated laparotomy and warn surgeons that we still do not know everything about the open abdomen.

\section{REFERENCES}

1. Rotondo MF, Schwab CW, McGonigal MD, Phillips GR 3rd, Fruchterman TM, Kauder DR, et al. 'Damage control': an approach for improved survival in exsanguinating penetrating abdominal injury. J Trauma. 1993;35(3):375-82; discussion 382-3. 